Proceeding of the $1^{\text {st }}$ ICEENG conference, 24-26 March, 1998.

\title{
ADAPTIVE NEURAL NETWORK FOR REAL-TIME
TRACKING CONTROL OF A DRIVE SYSTEM
}

\author{
R. S. Ahmed* , O. H. Abdalla*
}

\begin{abstract}
Neural Networks are attractive alternative to the classical techniques for identification and control of complex physical systems, because of their ability to learn and approximate functions. This paper presents the development and implementation of adaptive Multilayer Neural Network (MNN) controller in real-time for a drive system. A MNN is first trained off-line to learn (identify) the inverse dynamics of the system, after the training is successfully completed, the MNN is used as a feedforward controller in the control scheme.

The advantage of the proposed controller is that the MNN is permanently training. On-line learning is applied while the system is under control to capture any system parameter variations or disturbances. Simulation results are presented to show the advantages of adaptive MNN controller compared to nonadaptive MNN controller. Also, experimental results show that the adaptive MNN controller is able to control the speed trajectory of the drive system with a high degree of accuracy, even in the presence of disturbances.
\end{abstract}

\section{KEY WORDS}

Neural networks, DC motor, Real-time control, Tracking control, Interface board.

\section{INTRODUCTION}

Design of conventional control systems usually involves the development of a mathematical model of the system to derive a control law. In many of the physical systems, it may be difficult to obtain an accurate mathematical model due to the presence of structured and unstructured uncertainties. Therefore application of classical or adaptive control schemes to these systems may give unsatisfactory response for trajectory tracking control.

Instead of driving a controller via the controlled process mathematically, neural network controller methodology tries to establish the controller directly from input-output data of the controlled process without the need for its mathematical model $[1,2]$. An advantage of using artificial neural networks (ANNs) is their ability to learn nonlinear functions whose analytic forms are difficult to derive and whose solutions are burdensome to compute. ANNs can be trained with observed data from the real plant, to reproduce the characteristics of the plant without significant prior krowledge of the plant dynamics.

* Assistant Professor, ** Professor, Dept. of Elect. Eng.. Helwan University, Helwan, Egypt 11792. 
At present, there is an extremely strong interest in employing ANN techniques in several control application areas such as process control, robotics, vision and pattern recognition. One of the most common used neural network in the area of system identification and control is the feedforward multilayer neural networks (MNNs). Several studies have found that a feedforward MNN with sufficient number of hidden layer neurons can approximate any nonlinear continuos function to any desired accuracy through a learning algorithm $|3,4|$. Back-propagation learning algorithm is the most common used for training the feedforward neural networks [5,6]. The objective of neural networks based adaptive control system for unknown nonlinear plants is to develop algorithms for identification and control using ANNs through a learning process. Some research workers [7] used the neural network for identification and control of a drive system (DC motor) using a model reference adaptive control. Although good simulation results were obtained, the MNN controller was non-adaptive (fixed weights). Also, the MNN controller was not tested for disturbances and not implemented in real-time.

In this paper, an adaptive MNN controller is developed and implemented in real-time of a drive system (DC motor, interface circuits, sensors and load). The proposed control scheme does not require the system dynamic model and its parameters. A MNN is uscd to identify the system inverse dynamics. The MNN is first trained off-line using the input-output data obtained from the system hardware setup. After successfully training, the MNN is used as a feedforivard controller in the control scheme of the drive system. While the system is under control. on-line learning is used to update the weights of the MNN at each sampling instant to generate the appropriate control voltage for the drive system to follow a speed desired trajectory. Advantages of the adaptive MNN (using on-line learning) compared to nonadaptive MNN for controlling the: drive system are presented through simulation results

\section{NEURAL NETWORKS}

Artificial neural networks may be employed to represent the brain activities. The term artificial neural network is used to distinguish itself from the neural system in the human brain. There are many types of neural networks representing the brain structure and operation with various degrees of sophistication $[5,6]$. Artificial computing networks are far simpier than their biological counterparts becausc knowledge about actual brain functions is limited. No models have been successful in duplicating the performance of the human brain. Architecture of neural networks can be divided into two basic categories: feedforwrard, and feedback (recurrent) neural networks [6]. The feedforward neural network is commonly used for identification and control purpose

\subsection{Multilayer Feedforward Neural Network}

The multilayer feedforward neural network contains one input laver, one or two hidden layers and one output layer. A fully connected feedfe, rward MNN with one hidden layer is shown in Fig. 1. The hidden laver is connected to the input laver through the synaptic weights $\mathrm{V}_{\mathrm{i}}$, which form a matrix $\mathrm{V}$, and to the output laver through the synaptic iveights $w_{i k}$. which form a matrix $W$. The relation between the output value, $\mathrm{O}$, of the $\mathrm{k}^{\text {th }}$ output neuron and the inputs. $\mathrm{X}$. to the network is given by

$$
\mathrm{O}_{\mathrm{k}}=\mathrm{f}_{\mathrm{k}}\left[\sum_{\mathrm{j}=0}^{\mathrm{n}_{\mathrm{h}}} \mathrm{w}_{\mathrm{jk}} \mathrm{f}_{j} \sum_{\mathrm{i}=0}^{\mathrm{n}_{\mathrm{i}}} \mathrm{v}_{\mathrm{ij}} \mathrm{x}_{\mathrm{i}}\right] \text { for } \mathrm{n}_{\mathrm{0}} \geq \mathrm{k} \geq 1
$$

whe're $\mathrm{V}_{\mathrm{ij}}$ is the weight between the $\mathrm{j}^{\text {th }}$ hidden neuron and $\mathrm{i}^{\text {th }}$ input neuron, $\mathrm{W}_{\mathrm{j}} \mathrm{k}$ is the weight between the $\mathrm{k}^{\mathrm{th}}$ output neuron and $\mathrm{j}^{\text {th }}$ hidden neuron, $\mathrm{f}$ is the nonlinear activation function of the neurons, $\mathrm{n}_{\mathrm{i},} \mathrm{n}_{\mathrm{h}}$ and $\mathrm{n}_{\mathrm{l}}$ ars the number of in'put, hidden and output laver neurons. respectively 


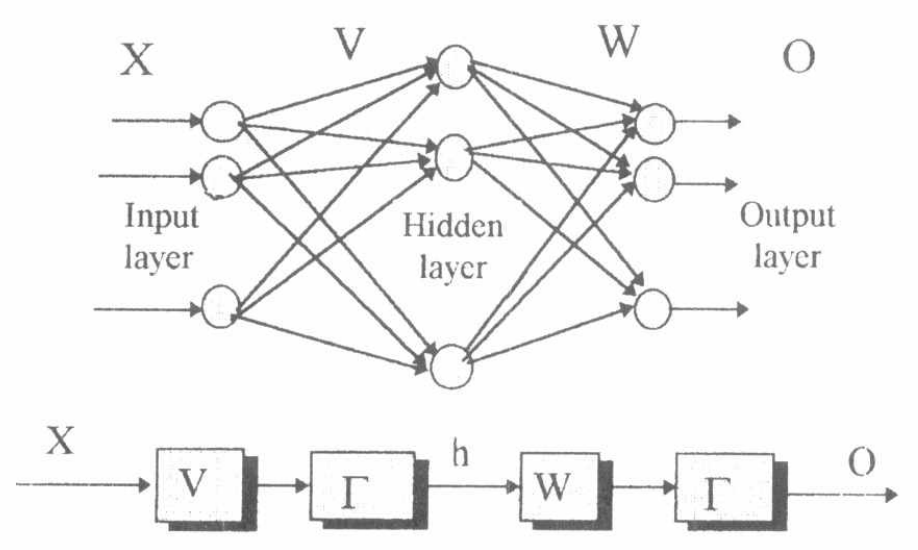

Fig. 1. A three layers feedforward neural network

The input-output mapping of a MNN can be represented by

$$
\mathrm{O}=\Gamma\left[\mathrm{W} \Gamma\left[\mathrm{VX}^{\mathrm{T}}\right]\right]
$$

where $\Gamma$ is a nonnlinear matrix operator. The transfer functions of the hidden $:$ nd output layer ncurons are, in general, similar and non-linear. If the transfer functions of the hidden layer neurons are linear, then the multilayer neural network is equivalent to a single-laver neural network

\subsection{MNN Learning}

Neural network learning (training) is the process of modifying the strength $\mathrm{c} f$ the connection weights between the network neurons. This modification leads to find an appropriate :et of weights so that the error between the desired and actual output of the network is minimized for ill training pattens. The network training starts by initially selecting small random weight values and pr sent ng all training data repeatedly. The learning algorithm may be categorized as: supervised learning and unsupervised learning. Supervised learning is used for learning the feedforward neural networks. In sujervised learning, a set of input and desired output patterns $(X, Y)$ called a training data set is required. The training data should be normalized (rescaled) to lie between $(0,1)$ or $(\because 1)$ der ending on the activation function of the neurons. At each instant of time when the input vector, $X_{i}$ is applied, the desired response of the system is provided by a teacher (desired output), $Y_{1}$. The error signal between the desired and the actual response is used to modify neural network parameters ('veights). An example of supervised learning is the backpropagation algorithm $[5,6]$.

Also there are two different schemes for training neural networks: In the first : pprcach which s called global (batch) learning, the weights are adapted after application of the whole input pattern vectors of the learning set. In the other approach referred to as local (pattern) learning, the weights are adapted after application of each pattern of the training set.

\subsection{Adaptive and Non-Adaptive MNN}

If the MNN is first trained (with a training sequence of finite length), and sulssequently used with the fixed weights obtained from training, this mode of operation is referrel to as "non-adaptive." Conversely, if the MNN is trained permanently while it is used (with a trai ning sequence of infinite length), this mode of operation is referred to as "adaptive" [8]. 
Proceeding of the $1^{\text {st }}$ ICEENG conference, 24-26 March, 1998.

\section{SYSTEM DESCRIPTION}

The simulation and experimental studies through this paper are done for a drive system composed of a permanent magnet DC motor, a power amplifier circuit to supply the power to the motor, a magnetic break for loading the motor and a tacho-meter to sense the motor speed.

\subsection{System Model}

The DC motor dynamics are given by the following equations

$$
\begin{aligned}
& \frac{\mathrm{di}_{\mathrm{a}}(\mathrm{t})}{\mathrm{dt}}=-\frac{\mathrm{R}_{\mathrm{a}}}{\mathrm{L}_{\mathrm{a}}} \mathrm{i}_{\mathrm{a}}(\mathrm{t})-\frac{\mathrm{K}}{\mathrm{L}_{\mathrm{a}}} \omega(\mathrm{t})+\frac{\mathrm{l}}{\mathrm{L}_{\mathrm{i}}} \mathrm{v}_{\mathrm{t}}(\mathrm{t}) \\
& \frac{\mathrm{d} \omega(\mathrm{t})}{\mathrm{dt}}=\frac{\mathrm{K}}{\mathrm{J}_{\mathrm{a}}} \mathrm{i}_{\mathrm{a}}(\mathrm{t})-\frac{\mathrm{D}}{\mathrm{J}_{\mathrm{a}}} \omega(\mathrm{t})-\frac{1}{\mathrm{~J}_{\mathrm{a}}} \mathrm{T}_{1}(\mathrm{t})
\end{aligned}
$$

where $\omega, \mathrm{V}_{\mathrm{t}}, \mathrm{i}_{\mathrm{a}}, \mathrm{R}_{\mathrm{a}}, \mathrm{L}_{\mathrm{a}}, \mathrm{J}_{\mathrm{a}}, \mathrm{K}, \mathrm{D}$ and $\mathrm{T}_{1}$ are the rotor speed, terminal voltage, armature current, armature resistance, armature inductance, rotor inertia. torque (back emf) constant, damping constant and load torque, respectively. The armature voltage. $V_{1}$ of the DC motor is supplied from the power amplifier circuit and is proportional to its control voltage $\mathrm{V}_{\mathrm{c}}$. If the relationship between $\mathrm{V}_{t}$ and $\mathrm{V}_{\mathrm{c}}$ is considered a linear, and from equations (2) and (3). the relationship between the control voltage and the motor speed can be written as a difference equation as

$$
\mathrm{V}_{\mathrm{c}}(\mathrm{k})=\beta \omega(\mathrm{k})+\gamma \omega(\mathrm{k}-1)+\zeta \omega(\mathrm{k}-2)
$$

where $\beta, \gamma$ and $\zeta$ are constants and their values depend on the motor parameters and the sampling interval T. Equation (4) can also be written in the following form

$$
\mathrm{V}_{\mathrm{c}}(\mathrm{k})=h(\omega(\mathrm{k}), \omega(\mathrm{k}-1), \omega(\mathrm{k}-2))
$$

The object of the system model is to find a relation between the system output and its input. This is useful in the next section's to determine the number of input and output layer neurons of the neural network controller

\subsection{System Hardware Setup}

The hardware setup of the drive system consists of a permanent magnet DC motor, power amplifier, magnetic brake for load conditions, tacho-generator for speed sensing and personal computer (PC). An interface board $(\mathrm{A} / \mathrm{I})$ and $\mathrm{D} / \mathrm{A}$ converters, and digital $\mathrm{I} / \mathrm{O}$ lines) is used to offer a data acquisition solution. A PC 48fj AT is used for implementation of the software neural network controller. The computer processor: exccutes the software controller program which is written in C language. Data transfer from and to the drive system is accomplished via the PC memory. The digital inputs from the system interface to the software controller are taken through the A/D converter, while the analog outputs from the software controller to the system interface are taken through D/A converter of the interface board 


\section{MNN IDENTIFICATION OF THE SYSTEM INVERSIE DYNAMICS}

A MNN is used to identify the unknown system dynamics (DC motor, amplifier circuit, serısor and load) that maps the control voltage $\mathrm{V}_{\mathrm{c}}$ to the motor speed $\omega$. Because the $\mathrm{MNN}$ is used to identify the inverse dynamics of the system, the inputs to the MNN is the system output (motor speed), and the output from the MNN is the control voltage. The MNN is trained to emulate the unkunwn function, $h(\bullet)$ in equation (5), so the inputs to the MNN are $\omega(\mathrm{k}), \omega(\mathrm{k}-1)$ and $\omega(\mathrm{k}-2)$ and the $M N V$ output, $V_{\mathrm{nn}}(\mathrm{k})$ is the estimate of the control voltage $\mathrm{V}_{\mathrm{c}}(\mathrm{k})$. The $\mathrm{MNN}$ consists of three layer $\mathrm{s}, 3$ neurons in $\mathrm{t}$ (c input layer, 7 neurons in the hidden layer and one neuron in the output luyer. The normalized training data obtained from the hardware setup is used to train the MNN of-line. The wights of the MNN were randomly initialized with a small values $( \pm 0.2)$. The error signal, $\mathrm{e}(\mathrm{k})$, bet ween the desired output, $\mathrm{V}_{\mathrm{c}}(\mathrm{k})$, and the network output, $\mathrm{V}_{\mathrm{nn}}(\mathrm{k})$, is used to update the nawork weights du ing the learning process through the back-propagation learning algorithm [5,6]. Figure 2 shows the configuration of the system identification using a MNN. The estimate of the system inverse dynamics is contained in th: MNN. A.fter the training of the MNN is completed, it is tested with a test data (not sontcined in the training data). The output of the MNN was found to be vervelose to the desired output.

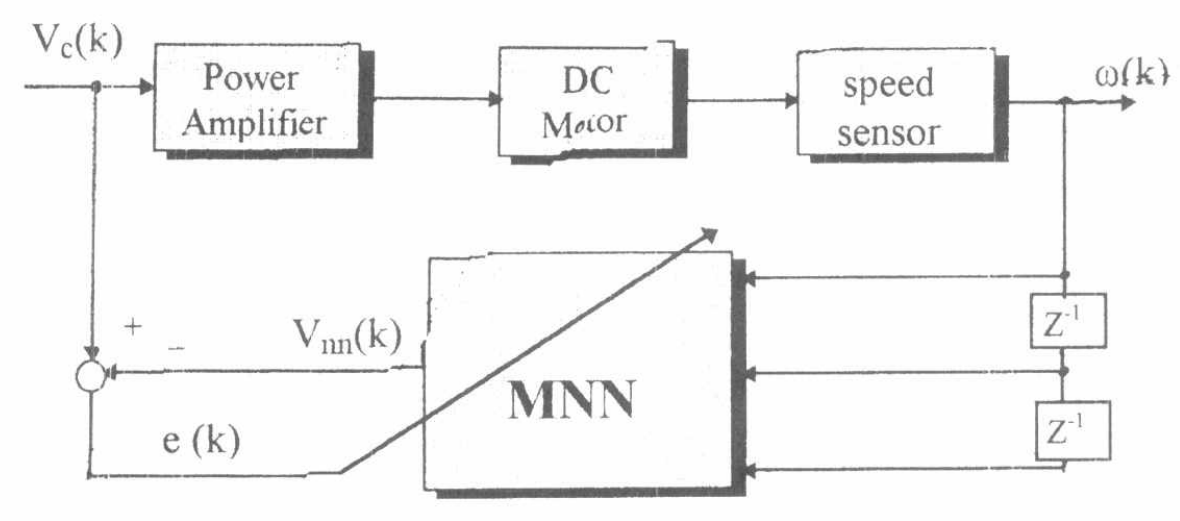

Fig. 2. System identification using a MNN

\section{ADAPTIVE MNN CONTROLLER}

The objective of the motor control system is to drive the motor so that its spe ad follow a pres jecified desired trajectory. The basic control scheme consists of the trained MNV which is use d as a feedforward controller and a fixed gain feedback controller as shown in Fig. 3. 4 fee:dback controller is used in the feedback loop to serve the on-line learning and to achieve low erre rs during transient. Online learning is used to tune the weights of the MNN to capture any variations of system parameters

and disturbances. The system control voltage, $\mathrm{V}_{\mathrm{c}}$. is composed of the out/rut of the feedionvard controller, $\mathrm{V}_{\mathrm{n} n}$, and the output of the feedback controller, $\mathrm{V}_{\mathrm{p}}$.

If the MNN is trained well, then the estimate and actual system inverse dynamic; are very close, and the MNN controller alone provides all the necessary voltage for the motor to track the desired speed trajectory and the output of the feedback controller will be almost zero. If ther: is a mismatch between the estimate of the system inverse dynamics and the actual system inverse dyna nics. on-line learning is used to adjust the weights of the MNN to generate the appropriate volta; re required for a desired trajectory. 


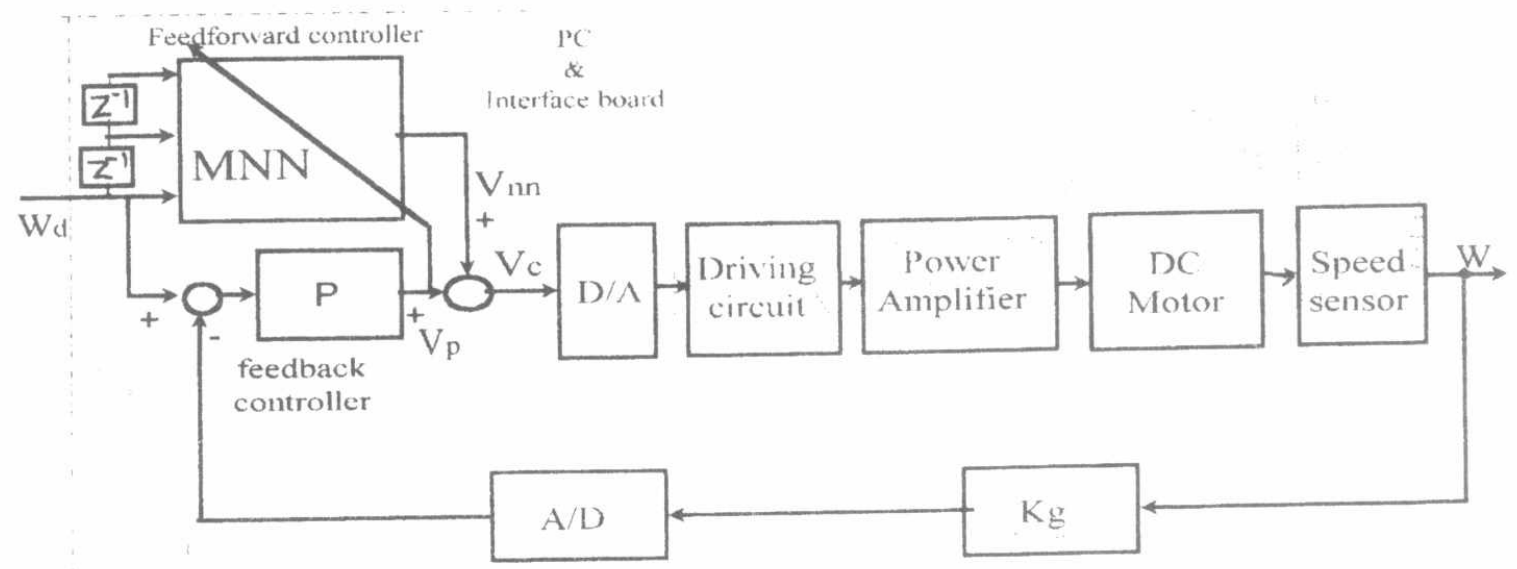

Fig. 3. Adaptive MNN controller for a drive system

\section{SIMULATION RESULTS}

Before implementing a real time controller, it is preferred, if possible, to cmploy a simulator rather than the actual process to be controlled. Dynamic simulations facilitate better understanding of the controlled dynamic process and provide insights into the nature of the interactions between the inputs and outputs In addition, performance of the proposed controller can be investigated by performing tests on dynamic simulators. So simulation results of the proposed adaptive MNN controller for the drive system is performed first. Also, the advantages of adaptive MNN controller compared to non-adaptive is presented through simulation results. Several motor speed trajectories were tested for adaptive and nonadaptive MNN controllers. For brevity, only the following trajectory is presented. Figure 4(a) shows the desired and simulated speed trajectories with non-adaptive (fixed weights) MNN controller when a sudden disturbance is applied at time $3.5 \mathrm{sec}$ and removed at $10.5 \mathrm{sec}$. The system control voltages are shown in Fig. 4(b). It can be seen that the actual system output does not follow the desired output very close. This is due to the fixed. weights of the MNN controller. To show the effectiveness of the on-line learning, the same previous trajectory with the same disturbance is repeated with adaptive MNN controller. Figure 5(a) shows the desired and simulated motor speed trajectories. The system control voltages in this case are shown in Fig. 5(b). It can be seen from this figure that the simulated and desired output trajectories are very close even in the case of disturbances.

\section{EXPERIMEN'TAL RESULTS}

The capability of the MNN controller for tracking control of the DC motor was tested in the laboratory by applying different speed trajectories. To ensure a robust performance, the control scheme was also tested for sudden clisturbances. The motor system was first tested without controller (open-loop). Figure 6(a) shows the desired and actual motor speeds when a desired constant speed of $1200 \mathrm{rpm}$ and a sudden disturbance is applied to the motor system at time $5.2 \mathrm{sec}$ and removed at $9.2 \mathrm{sec}$. It is shown from. this figure that the speed is dropped from $1200 \mathrm{rpm}$ to $780 \mathrm{rpm}$ during the period of disturbance A photograph of the actual motor speed and the control voltage is shown in Fig. 6(b)

Figure 7(a) shows the desired and actual motor speeds of the previous case with the adaptive MNN controller. It can be seen from these figures that the actual motor speed follows the desired speed very 


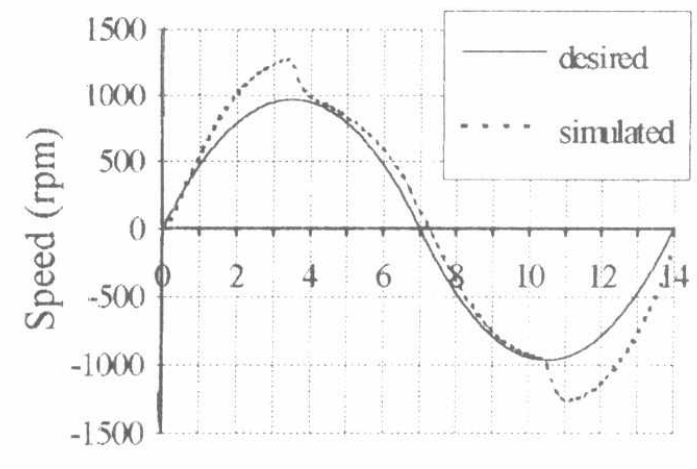

Time (sec)

Fig. 4(a). Desired and simulated speeds (non-adaptive MNN controller)

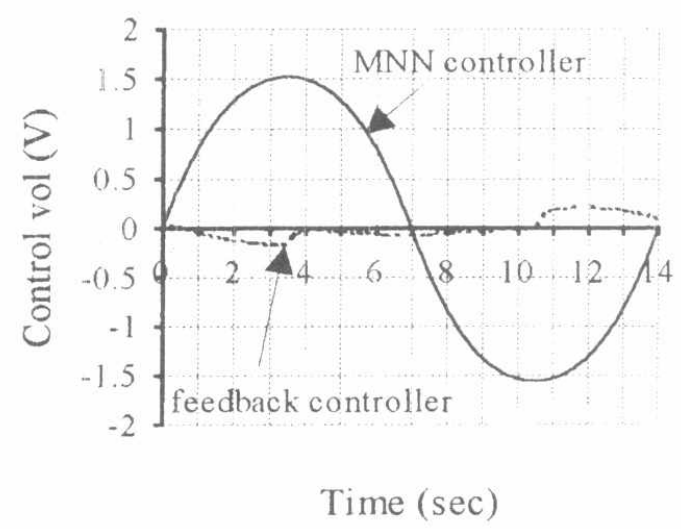

Fig. 4(b). System control voltages

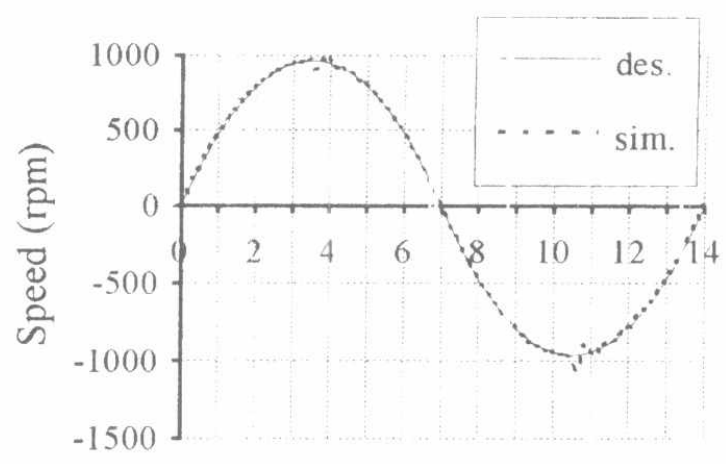

$\operatorname{Tims}(\mathrm{sec})$

Fig. 5(a). Desired and simu ated speeds (with adaptive MN'J controller)

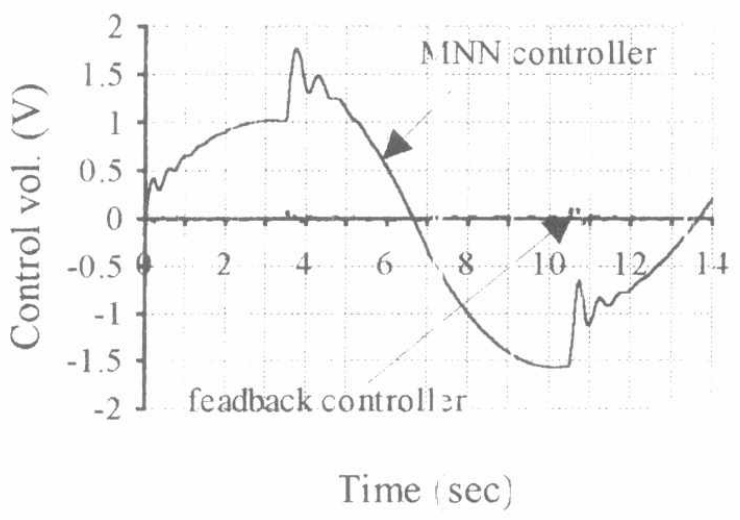

Fig. 5(b). Systen control voltages

closely, and the control system is able to handle the sudden disturbance appl ed to the motor system. The output of the MNN controller changes in response to the disturbance inp it whereas the output of the feedback controller is approximately zero except at the starting and at th: instant of applying the disturbance. The MNN controller provides almost all the voltage to the motor system to track the desired trajectory as shown in Fig. 7(b). A photograph of the actual motor speed and the control voltage is shown in Fig. 7(c).

The system response to a parabolic speed trajectory with disturbance applied at time $3.3 \mathrm{sec}$ and removed at time $10.3 \mathrm{sec}$ is shown in Fig. 8. It can be seen from Fig. 8(a) that the actual motcr speed follows the desired speed trajectory very closely. Figure 8 (b) shows the system control voltages: It can be seen from this figure that the MNN controller provides almost all the control input voltage to the motor. The control input voltage provided by the feedback controller is ver small compared to the MNN controller and changes at the instants of applying and removing the disturbance. A photograph of the actual motor speed, position and the control voltage is shown in Fig. 8(c). It can be seen that the actual motor speed trajectory follows the desired speed trajectory very wel It is shown from these results that the adaptive MNN controller is efficient for trajectory control of the lrive system. 


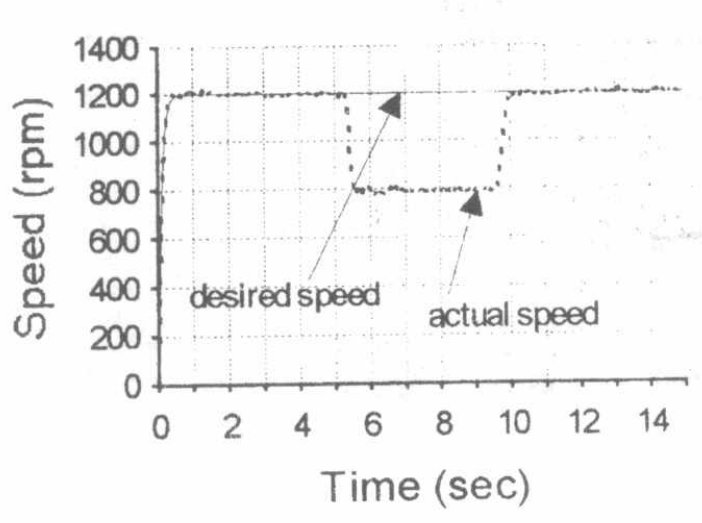

Fig. 6(a). Desired and actual motor speeds without controller.

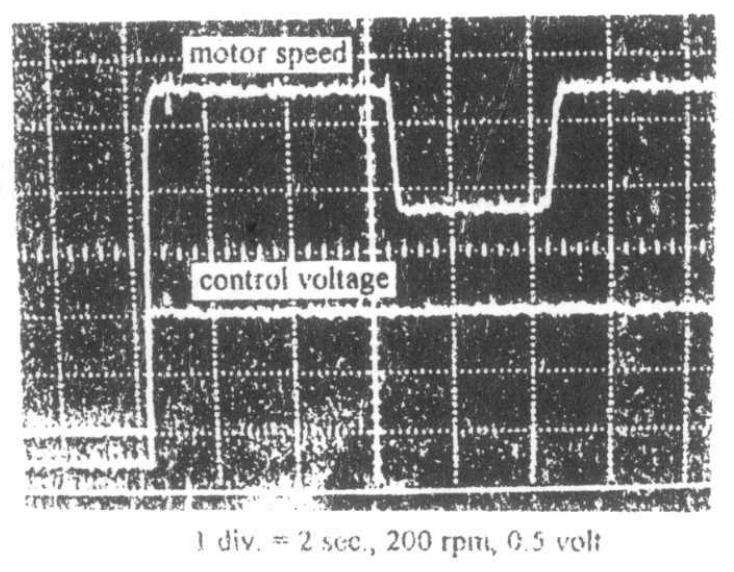

Fig. 6(b). Photograph of the actual motor speed and control voltage

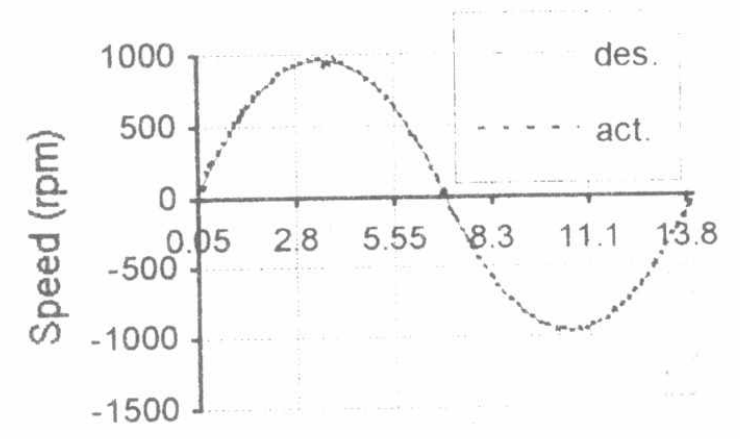

Time (sec)

Fig. 8(a). Desired and actual motor specds

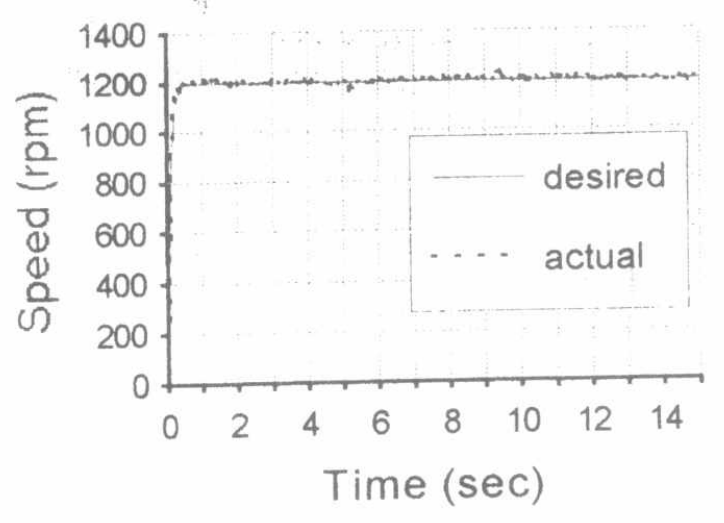

Fig. 7(a). Desired and actual motor speeds with MNN controller

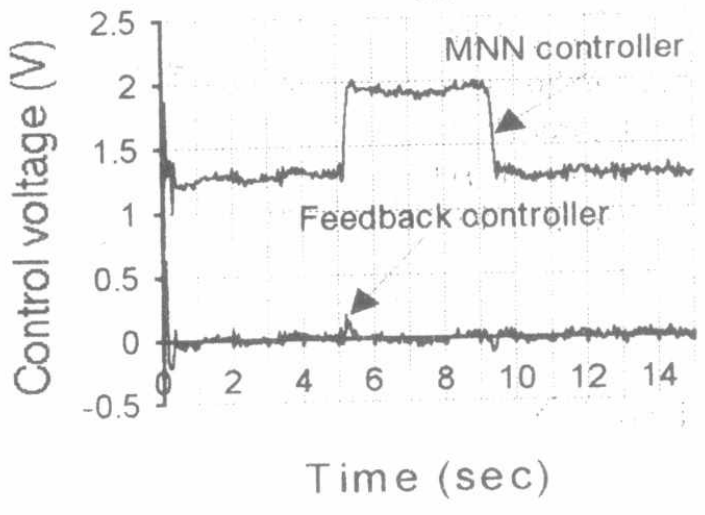

Fig. 7(b). Motor control voltages

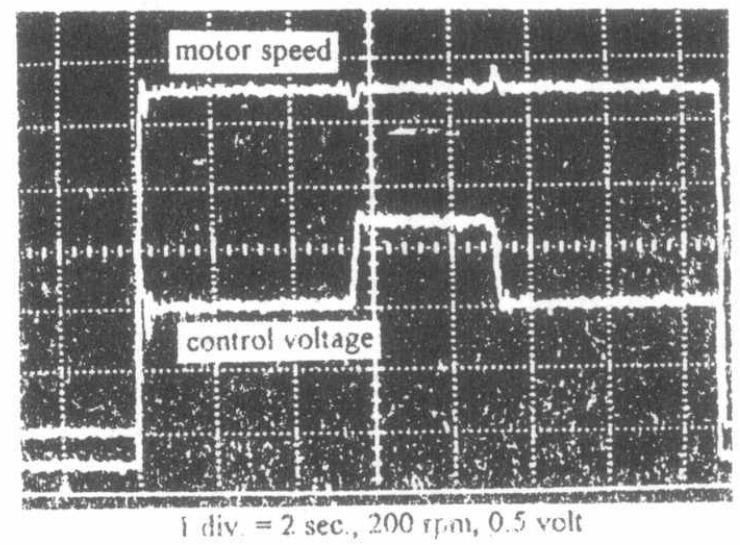

Fig. 7(c). Photograph of the actual motor speed and control voltage 


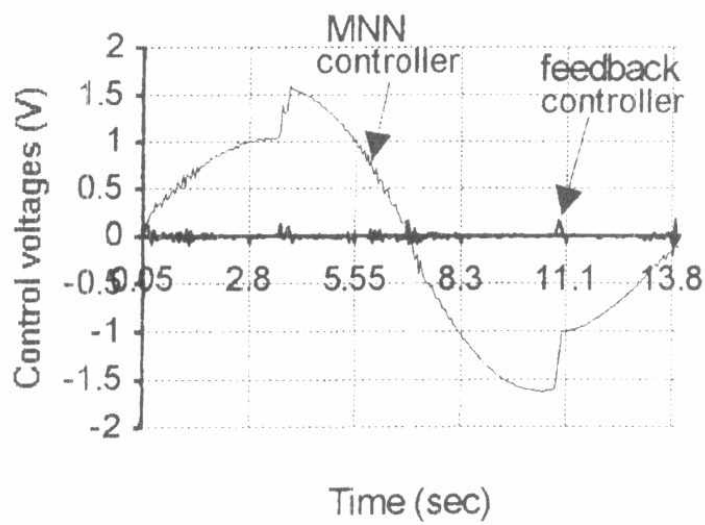

8(b). Motor control voltages

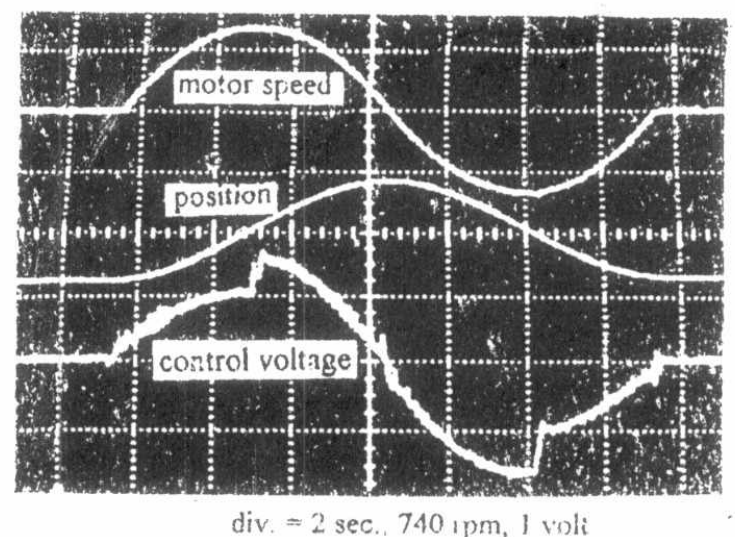

Fig. 8(c). Photograph o the actual motcr speed, position and co itrol voltage

\section{CONCLUSIONS}

This paper presents the development and implementation of a real-time adapti e MNN controller for a drive system (DC motor). The advantages of this scheme is that it does not req model and its parameters and therefore treats the system as a black box. W. Tile the system is under control, on-line learning is used to adjust the weights of the MNN to captur any system parameters variation and/or external disturbances. The advantages of the adaptive MN. N over non-adaptive is presented through simulation results. It is shown by simulation and experimental results that the proposed control scheme with the adaptive MNN is efficient for trajectory trac sing control of the drive system with high degree of accuracy even in the case of sudden disturbance.

\section{REFERENCES}

[1] Narendra K. S. and Parthasarathy K., "Identification and Control of D: namical Systems Using Neural Networks," IEEE Transactions on Neural Networks, vol. 1, pp. 4-i7, March 1990.

[2] Ahmed R. S., Rattan K. S. and Abdallah O. H., "Adaptive Neural Netivork For Identificat: on And Tracking Control Of A Robotic Manipulator," IEEE National Arrospace \& Elestronics Conference, Dayton, OH. pp. 601-609, May 1995.

[3] Bulm E. K. and Li L.K., "Approximation Theory and Feedforward Networks," Neural Networks, vol 4, pp. 511-515, 1991.

[4] Hornik W. K. and Stinchombe M., "Multilayer Feedforward Ne/works Are Uiiversal Approximators," Neural Networks, Vol. 2, pp. 359-366, 1989

[5] Zurada J. M., Introduction to Artificial Neural Systems, West Publishing Co npany, 1992.

[6] Haykin S., Neural Networks: A Comprehensive Foundation, Macmillan College, 1994.

[7] Weerasooriya S. and El-Sharkawi M. A., "Identification and Control of a DC Motor Using BackPropagation Neural networks," IEEE Trans. On Energy Conversion, Vinter Meeting, pp. 1-7, 1991.

[8] Nerrand O., et. al. "Training Recurrent Networks: Why and How? An I lustration in Dyıamical Process Modeling," IEEE Trans. On Neural Networks, Vol. 5, No. 2, March 1994. 